\title{
Cyclostationary analysis of fan noise influenced by an inflow control device
}

\author{
Larisa Grizewski* and Maximilian Behn ${ }^{\dagger}$ \\ German Aerospace Center (DLR), Berlin, Germany \\ Stefan Funke \\ Rolls-Royce Deutschland, Dahlewitz, Germany \\ Henri A. Siller ${ }^{\S}$ \\ German Aerospace Center (DLR), Berlin, Germany
}

Acoustic data measured in a fan test facility with and without an inflow control device (ICD) over the inlet were analyzed using the cyclostationary analysis technique, which separates the total power into a rotor locked and a fluctuating component. Measurements were taken with an inlet mode detection ring upstream of the rotor and with far-field microphones for configurations with subsonic and with supersonic fan blade tip speeds. The ICD reduces the fluctuating component and stabilizes the rotor locked component of the frequency spectrum at the blade passing frequency and its harmonics in the way that the mode amplitudes of the TylerSofrin modes increase, while neighboring mode orders are suppressed. The mode distribution and inter-mode coherence in the inlet are also changed, which changes the far-field directivity when an ICD is installed. It is demonstrated that cyclostationary analysis is a powerful tool to investigate the acoustic effect of an ICD on turbofan noise, revealing more details about the temporal and spatial structure of the generated sound field than a classical spectral analysis.

\section{Nomenclature}

$\begin{array}{ll}A_{m} & =\text { Azimuthal mode amplitude in } \mathrm{Pa} \\ \mathbf{a} & =\text { Mode amplitude vector in } \mathrm{Pa} \\ C_{m}^{\mu} & =\text { Inter-mode coherence of mode order } m \text { w.r.t. mode order } \mu \\ m & =\text { Azimuthal mode order } \\ p(t) & =\text { Measured sound pressure amplitude in } \mathrm{Pa} \\ \mathbf{p} & =\text { Sound pressure vector in } \mathrm{Pa} \\ p_{\text {fluct }}(\omega) & =\text { Fluctuating sound pressure spectrum in } \mathrm{Pa}^{2}\end{array}$

Presented as paper 2019-2719 at the 25th AIAA/CEAS Aeroacoustics Conference in Delft, The Netherlands, under the title Analysis of the influence of inflow distortions on turbofan rotor noise; received 28 April 2020; revision received 12 August 2020; accepted for publication 17 December 2020.

*Research engineer, German Aerospace Center (DLR), Institute of Propulsion Technology, Department of Engine Acoustics, Berlin, Germany

${ }^{\dagger}$ Research engineer, German Aerospace Center (DLR), Institute of Propulsion Technology, Department of Engine Acoustics, Berlin, Germany

\$Development engineer, Rolls-Royce Deutschland, Dahlewitz, Germany

${ }^{\S}$ Research scientist, German Aerospace Center (DLR), Institute Propulsion Technology, Department of Engine Acoustics, Berlin, Germany 


$$
\begin{array}{ll}
p_{\text {lock }}(\omega) & =\text { Rotor-locked sound pressure spectrum in } \mathrm{Pa}^{2} \\
p_{\text {tot }}(\omega) & =\text { Total sound pressure spectrum in } \mathrm{Pa}^{2} \\
\mathbf{S}_{p p} & =\text { Cross-spectral matrix of sound pressure in } \mathrm{Pa}^{2} \\
\mathbf{S}_{a a} & =\text { Cross-spectral matrix of mode amplitudes in } \mathrm{Pa}^{2} \\
\mathbf{W} & =\text { Mode transfer matrix }
\end{array}
$$

\section{Introduction}

The levels of spectral peaks at the blade passing frequency (BPF) of the fan radiated by turbofan engines increase when the flow entering the engine is distorted. Stationary, local variations of the flow into the engine, vorticity, and turbulence can increase the sound pressure levels at the blade passing frequency of the fan and its higher harmonics in the order of $10 \mathrm{~dB}$ or more. For engine acoustic ground tests, this is not acceptable and therefore inflow control devices (ICDs) that improve the quality of the inflow have been developed and have to be used for engine acoustic ground tests or fan noise tests according to ICAO regulations. SAE ARP1846 [1] prescribes the use of an ICD and its acoustic calibration and information on the design of ICDs is given in SAE AIR1935 [2]. While the name ICD is most commonly used, other names are used for devices that serve the same purpose and employ similar design criteria: ICS inflow control structure, TCS turbulence control screen or simply turbulence filter. There is a noticeable split in the naming conventions between those authors who emphasize inflow distortions and others who give turbulence an important rôle.

The development of ICDs has taken place in the 70s and 80s of the last century, when Boeing and Pratt\&Whitney cooperated in the development and testing of the JT9D engine for the Boeing 747 [3, 4]. In the same period, analytical and experimental work for acoustic measurements with engines in wind tunnels was published by NASA [5, 6] and General Electric [7]. In 1982, NASA held a workshop on Flight Effects on Fan Noise [8], where the results of wind tunnel and flight tests with the JT15D engine are reported. Here, some important requirements for low fan noise are stated. Apart from the use of ICDs, it is important to avoid flow disturbances in the inlet, upstream of the rotor, e.g. the wakes of probes in the inlet duct. A more recent study of engine noise during ground tests is concerned with engine test runs at airports [9]. All of these studies describe an increase of BPF levels between ground or wind tunnel tests and flight tests by as much as $12 \mathrm{~dB}$, which are most significant as long as the blade tip speeds remain subsonic [3]. The explanation given is that turbulent or vortical structures with transverse length scales in the order of the blade tip spacing create additional sound sources at the rotor blades. When the local relative Mach numbers of the fan blade tips are supersonic, the inflow distortions become insignificant because the buzz-saw noise sources, caused by shock waves at the blade tips, are a much stronger sound source mechanism than the interaction of wakes or turbulence with the fan blades [3]. Without an ICD, the buzz-saw noise mechanism sets in at slightly lower shaft speeds and the directivity pattern of the BPF tones is more uniform than with an ICD [5]. The reason for this is that without an ICD, a larger number of modes exhibit high levels in the inlet, while in the configuration with an ICD, a single mode dominates the 
mode spectrum. Woodward \& Glaser [5] argued that a high number of individual, incoherent sources generate these complex mode spectra, whereas Sofrin \& Cicon [10] demonstrated with a 2D non-uniform flow model that multiple modes can be generated by the refraction of sound waves. In the presence of non-uniform flow, the azimuthal mode orders $m$ do not represent the circumferential wavenumbers of the physical eigenmodes. This results in azimuthal mode spectra with multiple dominant modes and could be falsely identified as mode scattering, eventhough the generated physical mode is merely distorted in the circumferential direction. In a recent study, Moreau \& Guérin [11] adapted this approach for an analytical prediction model for fan buzz-saw noise. They take 3D duct modes into account and show that with a distorted inflow, the development of the mode spectrum in the axial direction leads to multiple dominant modes.

In free-field or wind tunnel tests without an ICD, the engine shaft frequency may even become instationary, resulting in instantaneous fluctuations of the levels and frequencies of the BPF tones and even the engine thrust [3].

The publications cited name three major mechanisms for inflow distortions in the engine inlet:

- ground vortices,

- distortions of the flow into the engine, and

- atmospheric turbulence.

A common model for the generation of noise by incoming turbulence or vorticity is that the structures are stretched in the accelerated flow towards the inlet and then successively sliced by the rotor blades. The resulting pressure fluctuations and instantaneous flow separation at the blades generate noise at the blade passing frequency and its harmonics [12, 13]. For wind tunnel tests, Woodward and Glaser [5] report that BPF levels were successfully reduced by boundary layer suction at the interface between the flow straightener and the engine inlet, which effectively removed distortions in the boundary layer over the inlet lip.

Adkins [9] states that some of the older publications overrate the impact of atmospheric turbulence and postulates that vorticity is more important. It may be that the earlier studies did not discriminate between turbulence and vorticity and did not pay too much attention to the structure and length scales of the inflow distortions. It could make a difference whether the distortions are spatially and temporally randomly distributed or whether they are fixed locally like wakes or vortices.

According to Gliebe [7], the length scales of atmospheric turbulent structures are in the order of magnitude of the size of the engine inlet itself and are therefore not able to interact with the fan blades as efficiently as small scale structures generated by ground vortices or flow separation in the boundary layer over the inlet lip. This is in line with the result of an analytic study by Ganz [14] who shows that the sound generation mechanism is most efficient when the structures have a transverse length scale in the range of a quarter of the fan blade tip spacing and a large streamwise length, so that they interact with a large number of blades as they pass through the fan.

Increased BPF levels due to inlet flow distortions can also occur in indoor engine test stands. When DLR and Rolls-Royce started to investigate the possibility to use indoor engine performance test stands for acoustic tests [15], 
higher BPF levels than in free-field tests with ICDs have been observed [16].

Sturm \& Carolus [17] identified the interaction of the rotor with large scale inflow distortions originating from a large anechoic chamber as the dominating source mechanism for a low-speed axial fan and demonstrated that the noise levels could be reduced by installing a hemispherical ICD.

The development of acoustic indoor measurement methods has sparked a new interest in the exact source mechanisms that make flow distortions raise the BPF levels. Although the studies found in the literature have provided practical solutions for ICDs to reduce BPF levels on outdoor test beds and have proposed several noise source mechanisms, it is still unclear how exactly inflow distortions increase the BPF levels in ground or indoor tests without ICDs. This lack of information has motivated the present study, where the cyclostationary averaging technique has been applied to data measured in a fan test facility in order to investigate the differences in the structure of the sound field with and without an ICD.

\section{Experiments}

In the fan test facility of AneCom AeroTest in Wildau, near Berlin, Germany, back-to-back tests with and without an ICD have been performed. The facility consists of a large anechoic chamber and the fan test rig. Figure 1 presents a photograph of the rig with the ICD mounted on the inlet.

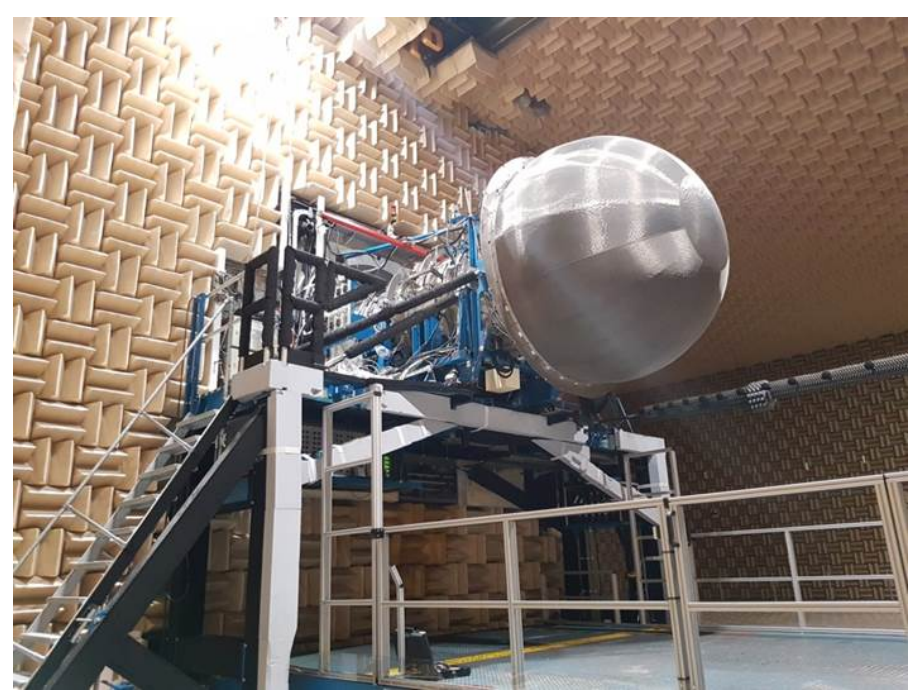

Fig. 1 The fan test rig of AneCom AeroTest with the ICD (source: AneCom AeroTest GmbH).

Measurement data were acquired using a mode detection ring in the inlet duct with 96 pressure sensors [18, 19], which are unevenly distributed over the circumference. Far-field acoustic data were measured with microphones on an arc around the inlet of the rotor test rig. The data presented were measured in two configurations: a low-speed configuration with subsonic fan blade tip speed, corresponding to an approach, and a high-speed, take-off configuration with supersonic fan blade tip speed. 
The measurements were performed with and without the ICD mounted over the inlet and between both sets of measurements, care was taken to adjust the corrected fan speeds to matching points on the working line. The fan speeds for the ICD were constant within $0,1 \%$ over the data acquisition period of $30 \mathrm{~s}$.

\section{Data analysis}

The data measured with the sensors in the mode detection ring and the far-field microphones were analyzed using cyclostationary averaging. Also, an azimuthal mode decomposition of the mode detection ring data was performed and the far-field directivity was determined for the blade passing frequency (BPF1) and its harmonic (BPF2).

The analysis consists of a cyclostationary averaging and separation of components with constant and random phase relation with respect to the rotor [20-22]. This method splits the total energy in one frequency band into a rotor locked and a fluctuating contribution. The rotor locked contribution is synchronous with the rotor shaft speed and represents the first order cyclostationarity, while the fluctuating component describes the energy with a random phase relation to the rotor in the same frequency band. The current analysis cannot distinguish higher order cyclostationarity for the fluctuating component [20].

\section{A. Cyclostationary data analysis}

The measured time series were resampled using a one-per-revolution trigger from the rotor shaft. The resampled time series are synchronous with the shaft rotation, irrespective of temporal variations of the absolute shaft frequency. For the spectral analysis, the resampled, rotor synchronous time series are subdivided into non-overlapping windows, which each contain the data of four successive rotations of the rotor shaft. Then, a complex fast Fourier transform (FFT) is performed for every window of the resampled time series. From the FFTs of the time signals, three different frequency spectra in engine order scaling can be derived by rotor synchronous averaging:

1) the total power spectrum $p_{\text {tot }}$, obtained by calculating the power of every FFT window $\mathcal{F}_{i}$ and averaging the results of all $N$ windows:

$$
p_{\text {tot }}(\omega)=\sum_{N} \frac{\left|\mathcal{F}_{i}(p(t))\right|^{2}}{N},
$$

2) the rotor locked component $p_{\text {lock}}$, which is the rotor synchronous part of the signal spectrum, obtained from averaging all complex FFT windows $\mathcal{F}_{i}$ and calculating the power of the result:

$$
p_{\text {lock }}(\omega)=\left|\sum_{N} \frac{\mathcal{F}_{i}(p(t))}{N}\right|^{2},
$$

3) the fluctuating component $p_{\text {fluct }}$, calculated from the complex FFTs by subtracting the complex average of all $N$ windows from every single FFT window $\mathcal{F}_{i}$, calculating the power of every single complex window, and then 
averaging over all $N$ windows:

$$
p_{\text {fluct }}(\omega)=\sum_{N} \frac{\left|\mathcal{F}_{i}(p(t))-\sum_{N} \frac{\mathcal{F}_{i}(p(t))}{N}\right|^{2}}{N}
$$

The cyclostationary analysis is based on averages of the data over FFT windows that contain the time series over several shaft rotations. The length of the averaging window, i.e. how many data points are included in the analysis, influences the frequency resolution as well as the ability to average over effects that may vary slowly with the shaft speed. The dependence of the results on the number of shaft rotations per FFT window was investigated by comparing results with FFT window sizes that were successively doubled between 2 and 64 rotations per FFT window. The window size influences the number of averages over the recorded number of rotor revolutions that can be calculated over a time series for a given rotor speed.

The averages of the total power and the dominating component are relatively stable against a doubling of the window size within a fraction of a decibel for the components that contain most of the energy, but may vary with the window size for the components that are more than $15 \mathrm{~dB}$ lower than the peak values. Here, a window size of 4 rotor revolutions was chosen in order to achieve between 500 and 1000 averages with an engine order resolution of 1/4 engine orders.

\section{B. Azimuthal mode decomposition}

The azimuthal mode decomposition of the inlet sound field, which was measured with the 96 dynamic pressure transducers in the mode detection ring array, is performed by use of the spatial Fourier transform:

$$
p(\varphi)=\sum_{m=-\infty}^{+\infty} A_{m} \cdot e^{i m \varphi} .
$$

In the case of the rotor locked components, a system of linear equations is formulated, which relates the complex sound pressure amplitudes $\boldsymbol{p}$ and the underlying azimuthal mode spectrum $\boldsymbol{a}$ :

$$
\boldsymbol{p}=\boldsymbol{W a},
$$

where $\boldsymbol{W}$ is a matrix containing the mode transfer functions $e^{i m \varphi_{k}}$ evaluated for the $m$ th mode order at the $k$ th sensor position. The mode spectrum is obtained by solving the system of linear equations in equation (5) using a Compressed Sensing technique as described in [23] for mode orders in the range from -79 to +79 , which is in the range that this array has been designed for by Rademaker et al. [18].

The fluctuating components are described statistically in terms of mean values and spatial cross-spectra. The spatial cross-spectrum is determined for each pair of sensors in the ring array. From equation 4 the relation between the mode 
amplitudes and the resulting sound pressure signal at the microphone array can be written in matrix notation as

$$
\mathbf{S}_{\mathrm{pp}}=\mathbf{W S}_{\mathrm{aa}} \mathbf{W}^{H}
$$

where $\mathbf{S}_{\mathrm{pp}}$ is the cross-spectral matrix of the pressure signals, $\mathbf{S}_{\mathrm{aa}}$ is the cross-spectral matrix of the mode amplitudes, and $\mathbf{W}$ the mode transfer matrix from equation 5 . The mode amplitudes of the fluctuating components are determined by solving equation (6) for the cross-spectral matrix $\mathbf{S}_{\mathrm{aa}}$ using the Compressed Sensing technique described in [23]. This technique reconstructs the complete cross-spectral matrix of the mode amplitudes, which enables the calculation of the inter-mode coherence between mode pairs $m$ and $\mu$ as

$$
C_{m}^{\mu}=\frac{\left|\left\langle A_{m} A_{\mu}^{*}\right\rangle\right|^{2}}{\left\langle\left|A_{m}\right|^{2}\right\rangle\left\langle\left|A_{\mu}\right|^{2}\right\rangle} .
$$

\section{Results}

Figure 2 illustrates the global effect of the ICD on measurements with the mode detection ring. It shows the spectra of the total power for the two configurations with and without the ICD for two different rotor speeds, one with subsonic and the other with supersonic fan tip speeds. Without the ICD, the low speed configuration features very high sound pressure levels at the BPF and its harmonics with broad bases around the peaks, similar to a haystacking effect. When the ICD is installed, the sound pressure levels of the BPF tones are substantially reduced by up to $14 \mathrm{~dB}$ for the BPF1 and the peaks become very sharp. The broadband noise levels between the BPF peaks remain at similar sound pressure levels.

The ICD changes the ranking of the BPF peaks: without the ICD, the BPF1 is the strongest peak and the sound pressure levels of the BPF harmonics decrease with the frequency such that the sound pressure level of BPF4 is about $4 \mathrm{~dB}$ below that of the BPF1 component. This balance is reversed when the ICD is in place: now the BPF1 is the weakest of the BPF harmonic peaks and the levels of the higher BPF harmonics increase with the frequency until BPF4 is almost $7 \mathrm{~dB}$ higher than BPF1.

The power spectra measured at the high rotor speed configuration with and without the ICD are dominated by multiple pure tone noise. The differences between the configurations with and without the ICD are not as substantial as in the low speed configuration. The BPF1 peak has almost the same sound pressure level with and without the ICD while the peaks at the higher BPF harmonics slightly increase when the ICD is installed. The spectral broadening is still visible in the data without the ICD, although it is less evident because of the multiple tonal peaks in the neighborhood of the BPF peaks. With the ICD, the broadband noise contribution, i.e. the sound pressure levels between the engine order peaks, is lower. Also, the distribution of the buzz-saw peaks changes when an ICD is installed. 

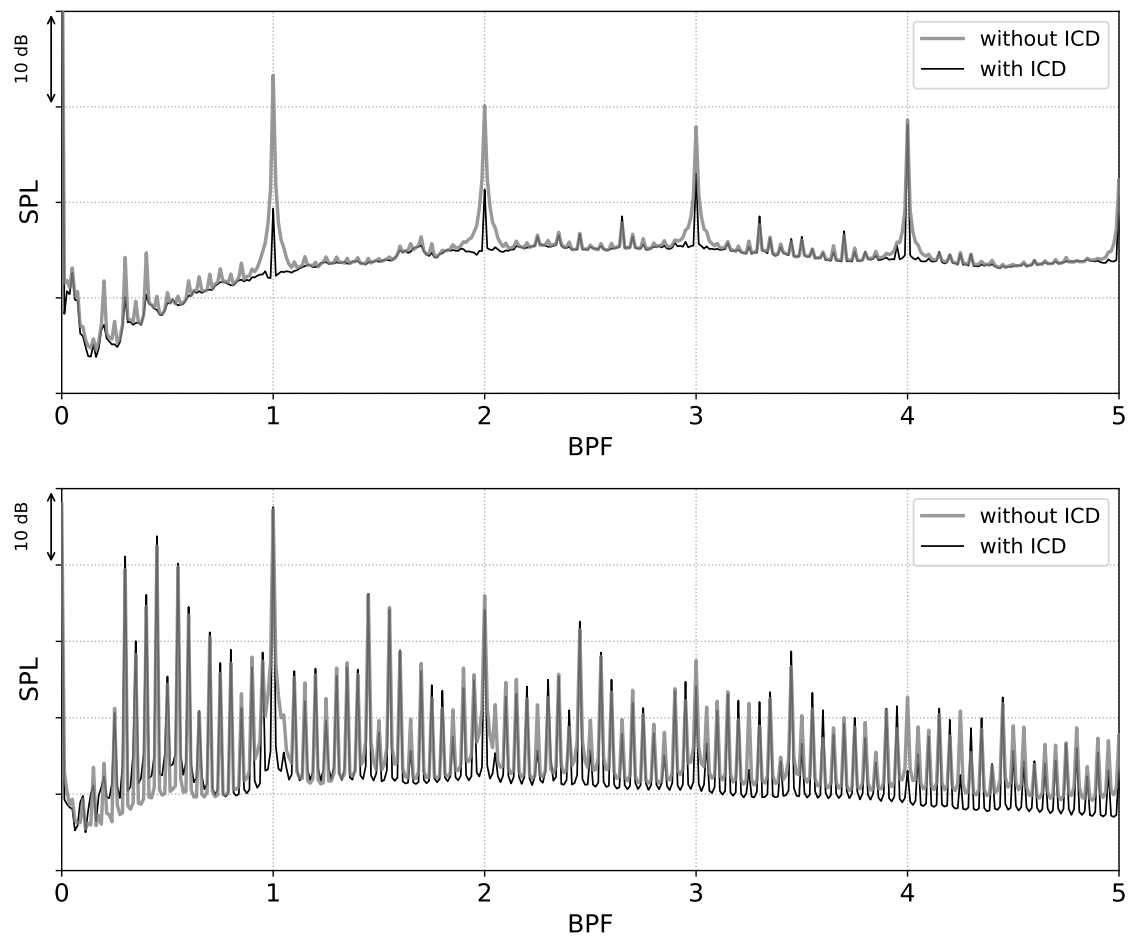

Fig. 2 Power spectra of the total power averaged over all sensors in the mode detection ring in engine order scaling for the configurations with low (top) and high (bottom) shaft speeds with and without an ICD.

The ICD has a substantial effect, especially on the levels of BPF tones at low shaft speeds. It reduces the levels of the fluctuations and removes the spectral broadening at the bases of the BPF tones. A further analysis based on cyclostationary averaging and a separation of the rotor locked and the fluctuating components will help to gain a better understanding of how the acoustic field and the sound sources are modified by an ICD. For the data analysis, frequency spectra in engine order scaling for the total power, the rotor locked, and the fluctuating components have been calculated from the data of the mode detection ring and the far-field microphones.

\section{A. Low shaft speed}

Figure 3 presents the spectrum of the total power and the rotor locked and fluctuating components measured in the inlet duct for the configuration with low, subsonic, rotor blade tip speeds, averaged over all sensors in the mode detection ring. In the configuration without the ICD, shown in the graph at the top of Fig. 3, the BPF1 is the strongest peak. The BPF1 peak is dominated by the fluctuating component, as shown in the zoom into the engine orders around BPF1 that is inserted in Fig. 3 The peak of the fluctuating component at BPF1 tapers off widely into neighboring engine order bands. The rotor locked component is weaker than the fluctuating component, but the peaks at the different engine orders are very sharp.

The graph on the bottom of Fig. 3 presents the spectrum of the total power and the rotor locked and fluctuating 

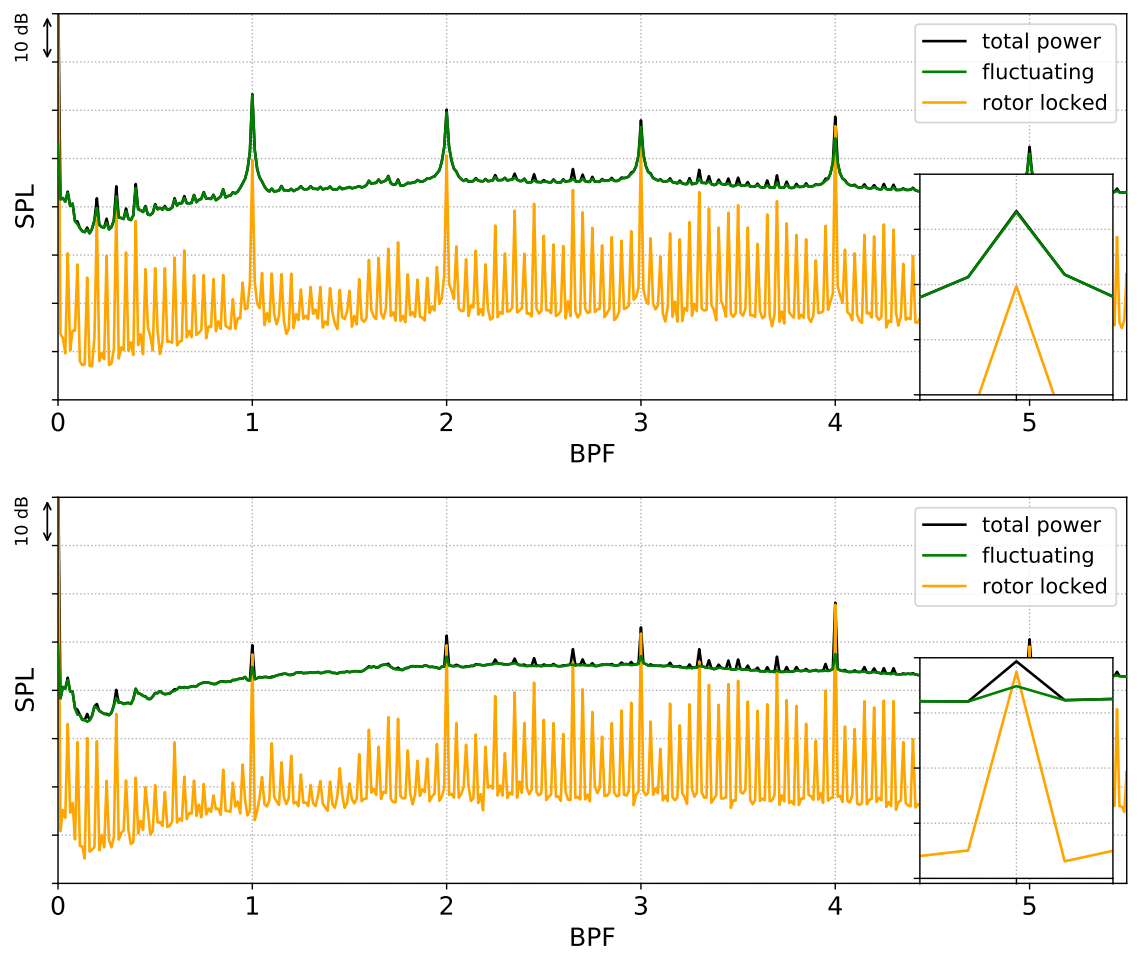

Fig. 3 Frequency spectra of the total power, the rotor locked, and the fluctuating component for the low-speed configuration, averaged over all pressure sensors in the mode detection ring. Top: without, bottom: with the ICD. The inserts show a zoom around the BPF1 peak.
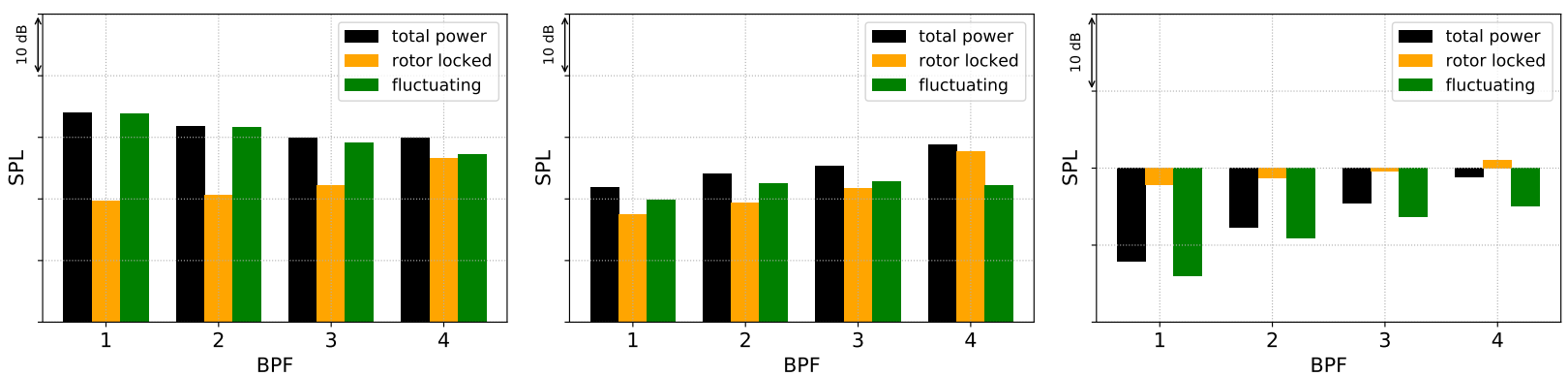

Fig. 4 Sound pressure levels of the BPF and its harmonics of the total power, the rotor locked, and the fluctuating components averaged over all sensors in the mode detection ring for the low-speed configuration. Left: without, centre: with the ICD, right: difference between both configurations.

components when the ICD is installed for the low speed configuration. The overall power at the BPF1 is reduced by about $14 \mathrm{~dB}$ compared to the case without the ICD (see Fig. 22). The reduction of the total power is caused mainly by the substantial reduction of the fluctuating component, which dominates the energy in the configuration without the ICD.

Figure 4 presents the levels of the total power, the rotor locked and fluctuating components in the frequency bands of the BPF and its harmonics, integrated over $\pm 0,5$ engine orders and averaged over all sensors in the mode detection ring. The levels of the BPF1, 2, 3, and 4 peaks are shown for the two configurations with and without the ICD, as well as the differences between both configurations. If the bars in the plot extend above the centre line, the ICD causes 
an increase of energy, if they reach below the centre line, the ICD reduces the energy of the respective component. The differences between the configuration with and without an ICD are large for the total power and the fluctuating component, but relatively weak for the rotor locked component, which is slightly reduced at BPF1, BPF2, and BPF3 and slightly increased at BPF4.

The effect of the ICD on the rotor locked and fluctuating components of the frequency spectra measured in the far field and averaged over all far-field positions is presented in Fig. 5. The spectra are qualitatively similar to those measured in the inlet duct, which confirms that the effect of the ICD is coupled to the sources on the rotor blades and is not substantially changed by propagation effects outside of the duct. The sound pressure levels at the BPF peaks, integrated over all far-field microphone positions, with and without the ICD are shown in Fig. 6. There are some differences in detail compared to the BPF levels in the inlet duct from Fig. 4, but the global effects are similar: without the ICD, the total power at the BPF harmonics decreases with the frequency and is dominated by the fluctuating component. The installation of an ICD reduces the total power at the BPF1 substantially, but also at the higher BPF harmonics and changes the balance of power between the BPF harmonics: with the ICD, BPF1 is weaker than BPF2 and BPF3. The relative contribution of the rotor locked component to the total power is significantly higher with the ICD, because the ICD substantially reduces the power of the fluctuating component.
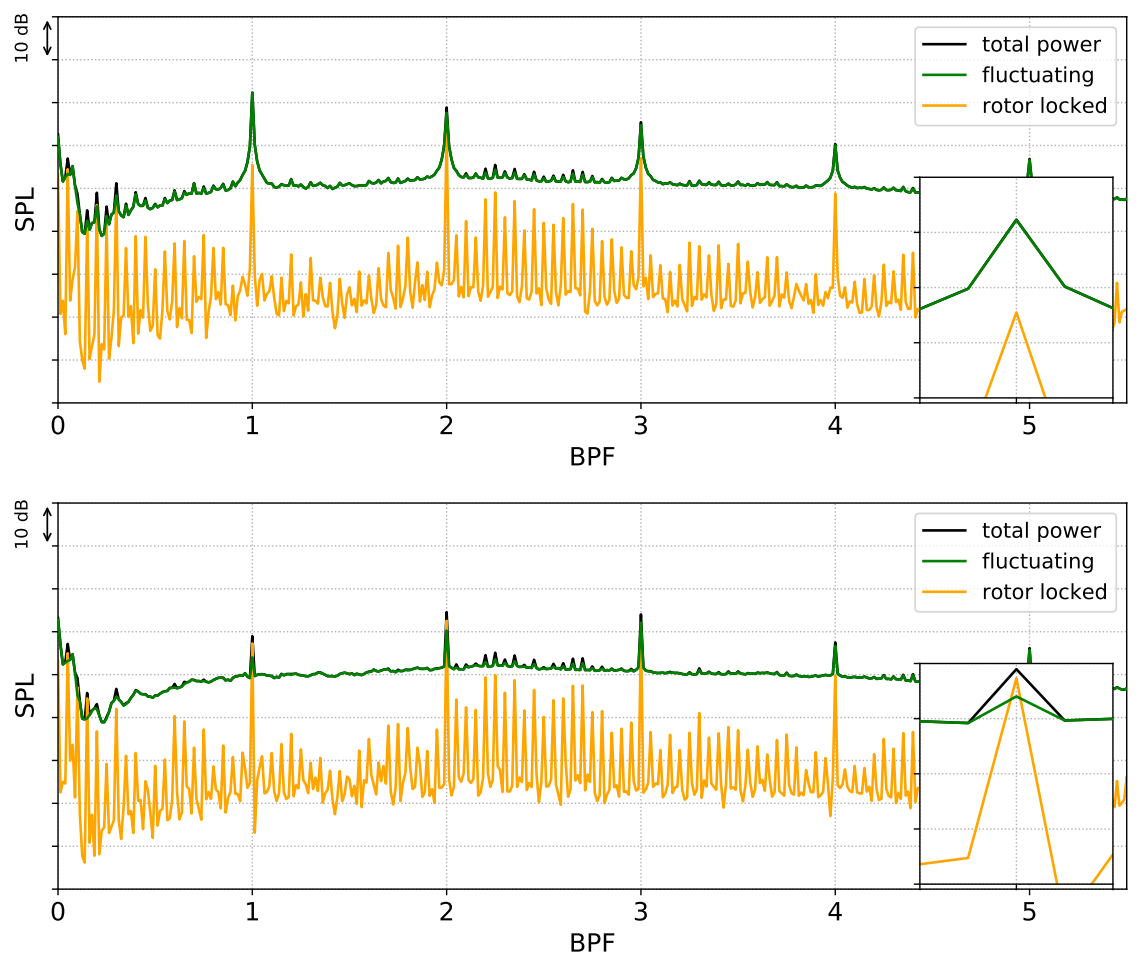

Fig. 5 Frequency spectra of the total power, the rotor locked, and the fluctuating component for the low-speed configuration, averaged over all microphones in the far-field microphone array. Top: without, bottom: with the ICD. The inserts show a zoom around the BPF1 peak. 

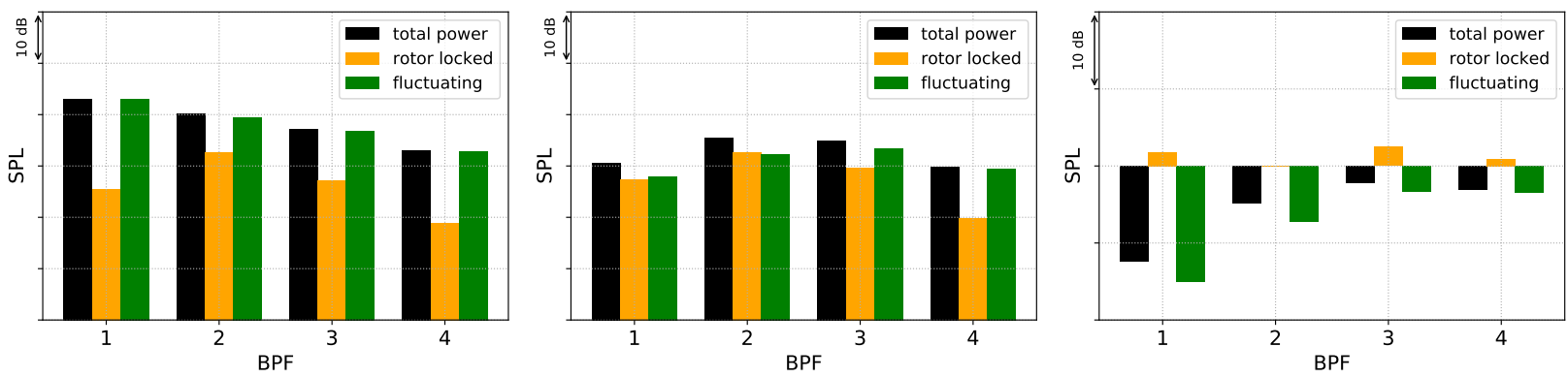

Fig. 6 Sound pressure levels of the BPF and its harmonics of the total power, the rotor locked, and the fluctuating components averaged over all microphones in the far-field microphone array for the low-speed configuration. Left: without, centre: with the ICD, right: difference between both configurations.
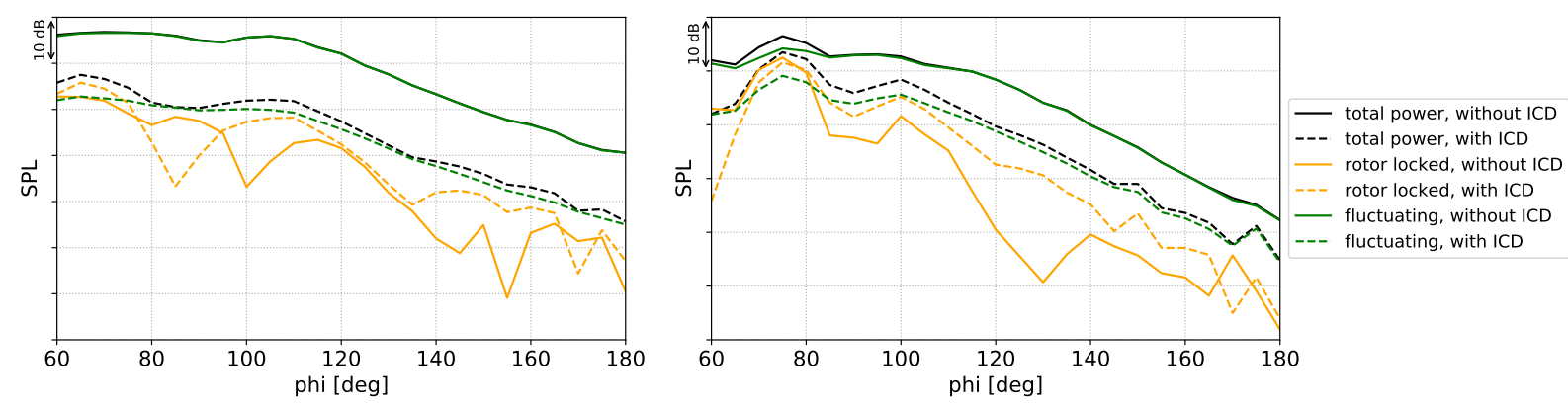

Fig. 7 Far-field directivity of the total power, the rotor locked, and the fluctuating components at the BPF1 (left) and BPF2 (right) for the low-speed configuration with and without an ICD. $180^{\circ}$ indicates the direction of flight.

The directivity of the BPF1 measured in the far field is presented in Fig. 7. The installation of the ICD lowers the total power and has a small effect on the directivity of the total power, by increasing the amplitudes of the lobes at $75^{\circ}$ and $90^{\circ}$. This is mainly caused by a net increase of the rotor locked component in these directions. The fluctuating component, which contributes almost all the energy in the configuration without an ICD, is reduced by over $10 \mathrm{~dB}$ in all directions and its directivity becomes flatter when the ICD is installed. The same trends can be seen in the directivity of the BPF2 shown in Fig.7 where the effect of the ICD on the directivity of the rotor locked component is similar and where the directivity of the fluctuating component is more pronounced than for BPF1.

Figure 8 presents the results of the mode analysis at BPF1 of the rotor locked and the fluctuating components, respectively, for the low speed experiments. The rotor locked part of the mode spectrum has a limited number of propagating modes, with levels of about $10 \mathrm{~dB}$ above the non-propagating modes. The modal distributions with and without the ICD are qualitatively similar, however they differ in detail: in both configurations, there is one mode on the left-hand side of the spectrum, co-rotating with the rotor, that is at least $5 \mathrm{~dB}$ above all the other modes, causing the lobed directivity pattern of the rotor locked component in the far field. The effect of the ICD on the modal content of the fluctuating component (see the right hand side graph in Fig. 8) is much more pronounced: the energy of all modes is reduced by more than $20 \mathrm{~dB}$. 

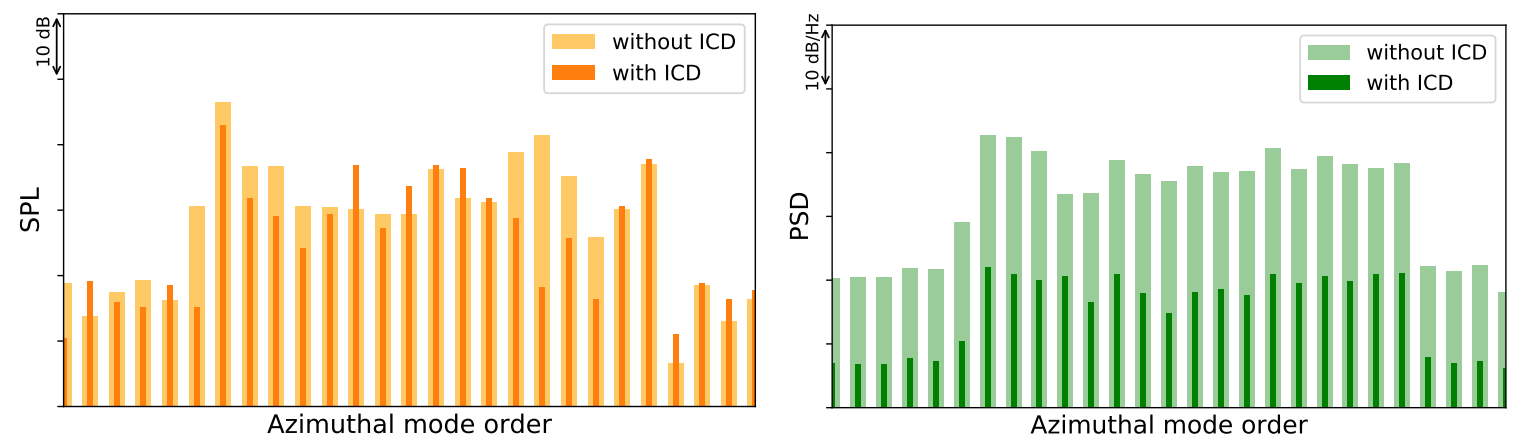

Fig. 8 Comparison of the mode distribution of the rotor locked (left) and the fluctuating (right) component at BPF1 for the low-speed configuration with and without the ICD.
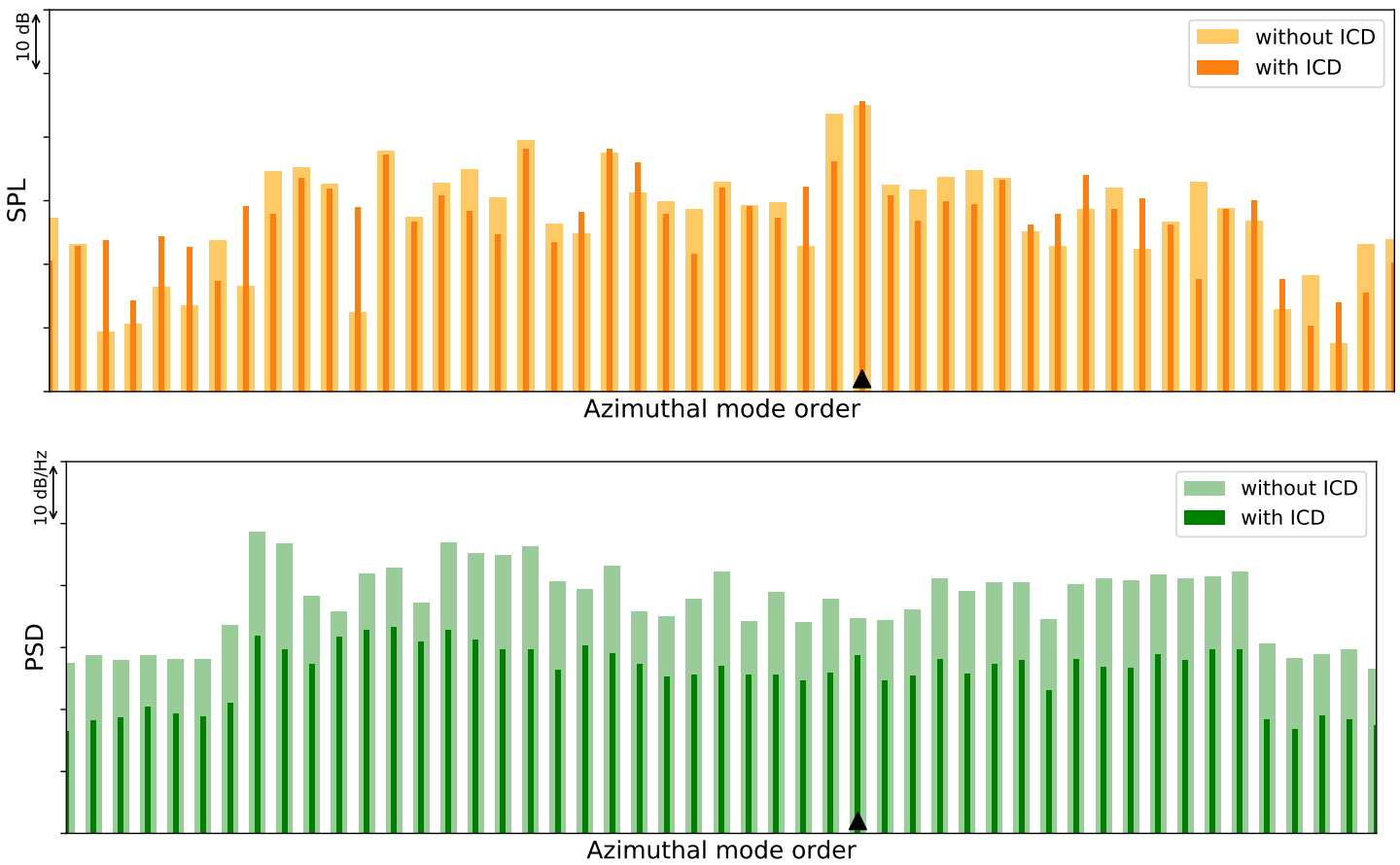

Fig. 9 Comparison of the mode distribution of the rotor locked (top) and the fluctuating (bottom) component at BPF2 for the low-speed configuration with and without the ICD. The black triangle marks the Tyler-Sofrin mode. 
The effect of the ICD on the azimuthal mode distribution of the BPF2 is shown in Fig. 9 for the rotor locked and the fluctuating components. The mode distributions are similar and as unspectacular as those of the BPF1, except for the feature that the Tyler-Sofrin mode is cut-on for BPF2. In the rotor locked component, the Tyler-Sofrin mode becomes more prominent when the ICD is in place because the neighboring modes are reduced. In the fluctuating component, all mode amplitudes are substantially reduced by the ICD, especially the co-rotating modes on the left hand side. In the fluctuating component, the Tyler-Sofrin mode is insignificant in the configuration without the ICD. With the ICD, it is not reduced by as much as the other modes and becomes one of the stronger modes.

The values of the inter-mode coherence, calculated relative to the mode with the highest amplitude for the fluctuating components at BPF1 and BPF2, are presented in Fig. 10. This analysis is only possible with cyclostationary averaging, because the highly coherent rotor locked components distort the results when the rotor-locked and fluctuating components of the signal are not separated. Without the ICD, the neighboring mode orders are partly coherent with the strongest mode, because the mode shapes are distorted by refraction in the non-uniform inflow, an effect that has been described by Sofrin \& Cicon [10]. This effect is even stronger at BPF2 than at BPF1. The homogenization of the inflow when an ICD is installed substantially reduces the coherence between neighboring modes. However, apart from neighboring mode pairs, inter-mode coherence is negligible for almost all mode pairs in both configurations.
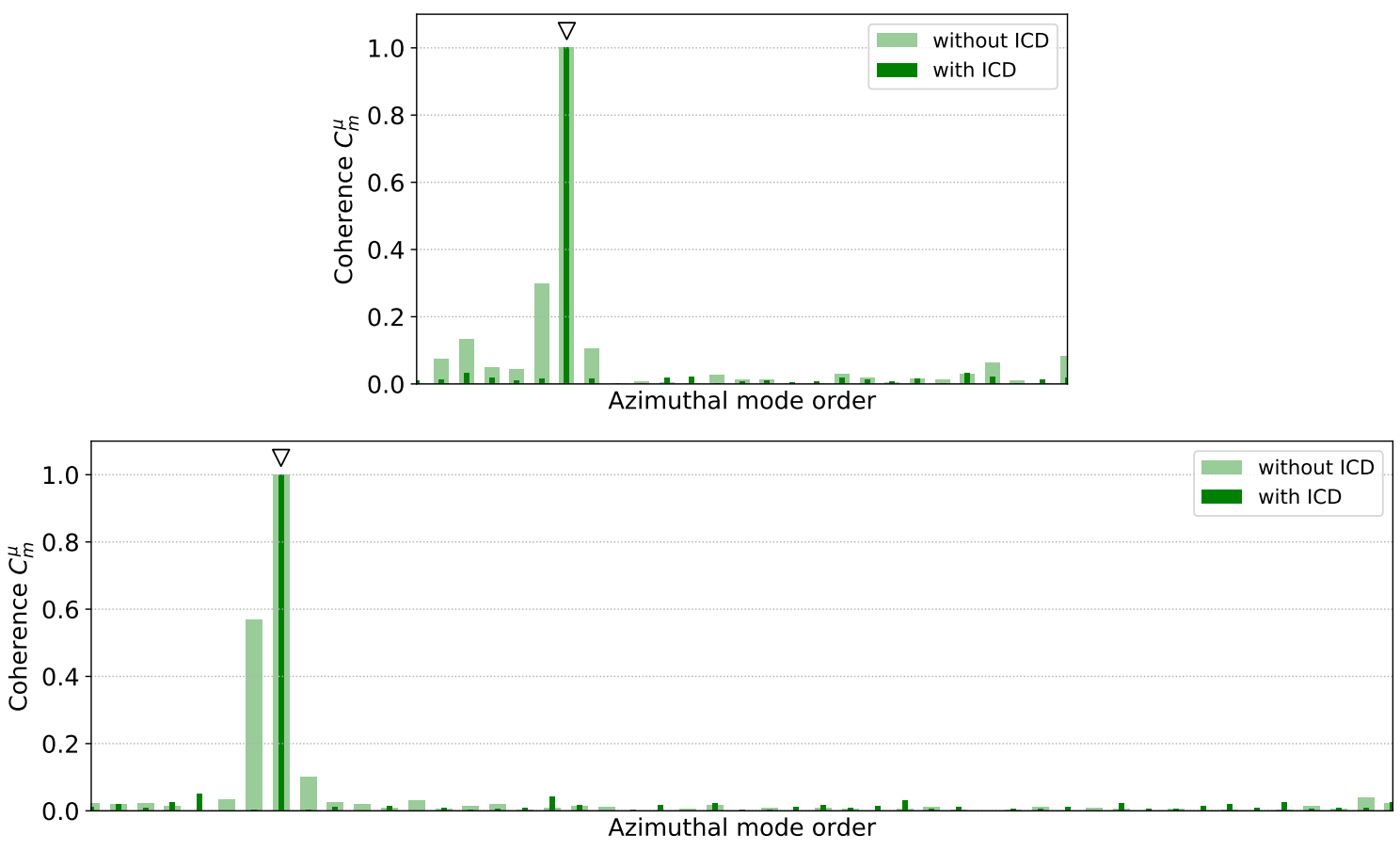

Fig. 10 Comparison of the mode coherence distribution of the fluctuating components w.r.t. the strongest mode (indicated by the black, hollow triangle) at BPF1 (top) and BPF2 (bottom) for the low-speed configuration with and without the ICD.

Both the mode amplitude and the mode coherence distribution influence the directivity in the far field. This has also 
been observed by Woodward et al. [5], who could only analyze the total power and could not separate the rotor locked from the fluctuating components.

The directivity of the rotor locked components shown in Fig. 7has a lobe structure with and without an ICD because in both cases, the mode spectrum has a low number of highly coherent modes that are substantially stronger than the others. The fluctuating component has a relative uniform directivity because of the large number of incoherent modes with similar amplitudes. The directivity of the total power is therefore more homogeneous in the configuration without an ICD, because the energy is dominated by the fluctuating component. With the ICD, the rotor locked component contributes significantly to the total energy and changes the directivity in the far field. This can be observed in Fig. 7 for BPF1, but it is even more pronounced at BPF2, where the Tyler-Sofrin mode becomes more important.

\section{B. High shaft speed}

Figure 11 presents the effect of the ICD on the total power spectrum, the rotor locked and the fluctuating components in the mode detection ring data at high rotor blade tip speed, averaged over all sensors of the mode detection ring. The configuration without the ICD is shown at the top and the configuration with the ICD on the bottom of the figure. In both configurations, the BPF1 is the strongest peak and the higher BPF harmonics reach successively lower sound pressure levels until BPF4.

The ICD does not change the ranking of the BPF peaks, but the sound pressure levels of the fluctuating component at the BPFs. The insert with the zoom around BPF1 in the top figure shows that without the ICD, the fluctuating and rotor locked components have similar levels. The bottom graph in Fig. 11 shows, that the ICD strongly decreases the levels of the fluctuating component, so that the rotor locked component dominates the BPF1 level.

Figure 12 illustrates this trend. The levels of the BPFs, integrated over $\pm 0,5$ engine orders and averaged over all sensors in the mode detection ring, are shown for the two configurations with and without ICD, together with the differences between both configurations. The ICD has a minor effect on the total power level at BPF1. However, the higher BPF harmonics are successively reduced by up to $7 \mathrm{~dB}$ at BPF4. The fluctuating components strongly decrease and the rotor locked components increase by a small amount.

The effect of the ICD on the total power spectrum, rotor locked and fluctuating components from the far-field data, averaged over all microphones in the arc, is shown in Figs. 13 and 14 . The spectra are qualitatively similar to those measured in the inlet duct, so there is no substantial propagation effect outside the duct, just like in the low-shaft-speed configuration. There are some differences in detail, but the global effect of the ICD on the BPF levels is still the same: the fluctuating component is strongly decreased, but not as strong as inside the duct. The levels of the rotor locked component increase, but substantially stronger than inside the duct, while the total power levels of the BPF and its harmonics are reduced in a similar way as in the in-duct measurements.

The far-field directivity of the BPF1 and BPF2 is presented in Fig. 15. At BPF1, the ICD reduces the total power 
level from polar angles of $60^{\circ}$ up to $118^{\circ}$, but increases it at higher angles (in the direction of flight). The total power in the forward arc increases by several decibels due to the increase of BPF1 levels between $118^{\circ}$ and $180^{\circ}$. Without the ICD, the total power level is dominated by the fluctuating component for polar angles up to $100^{\circ}$. Above $100^{\circ}$, the rotor locked component increases and starts to increase the total power level. The ICD reduces the fluctuating component strongly and the total power level is dominated by the rotor locked component over the entire angular range.

The directivity pattern of the rotor locked component is not significantly changed. For far-field radiation angles above $100^{\circ}$, the rotor locked component becomes even stronger than in the configuration without an ICD, which leads to a net increase of the rotor locked component in the far field when the levels are integrated over all directions, just like in the bar plots shown in Fig. 14. The graph on the right hand side of Fig. 15 shows similar tendencies for BPF2, with larger differences in the levels and angles of the radiation lobes.

Figures 16 and 17 present the results of the azimuthal mode analysis at BPF1 and BPF2 of the rotor locked and fluctuating components for the high rotor blade tip speed experiments. For both BPFs, there are strong differences between the results with and without the ICD. The effect of the ICD on the modal content of the rotor locked component as well as of the fluctuating component is very pronounced: the amplitudes of most modes are strongly reduced, for the fluctuating component stronger than for the rotor locked component.
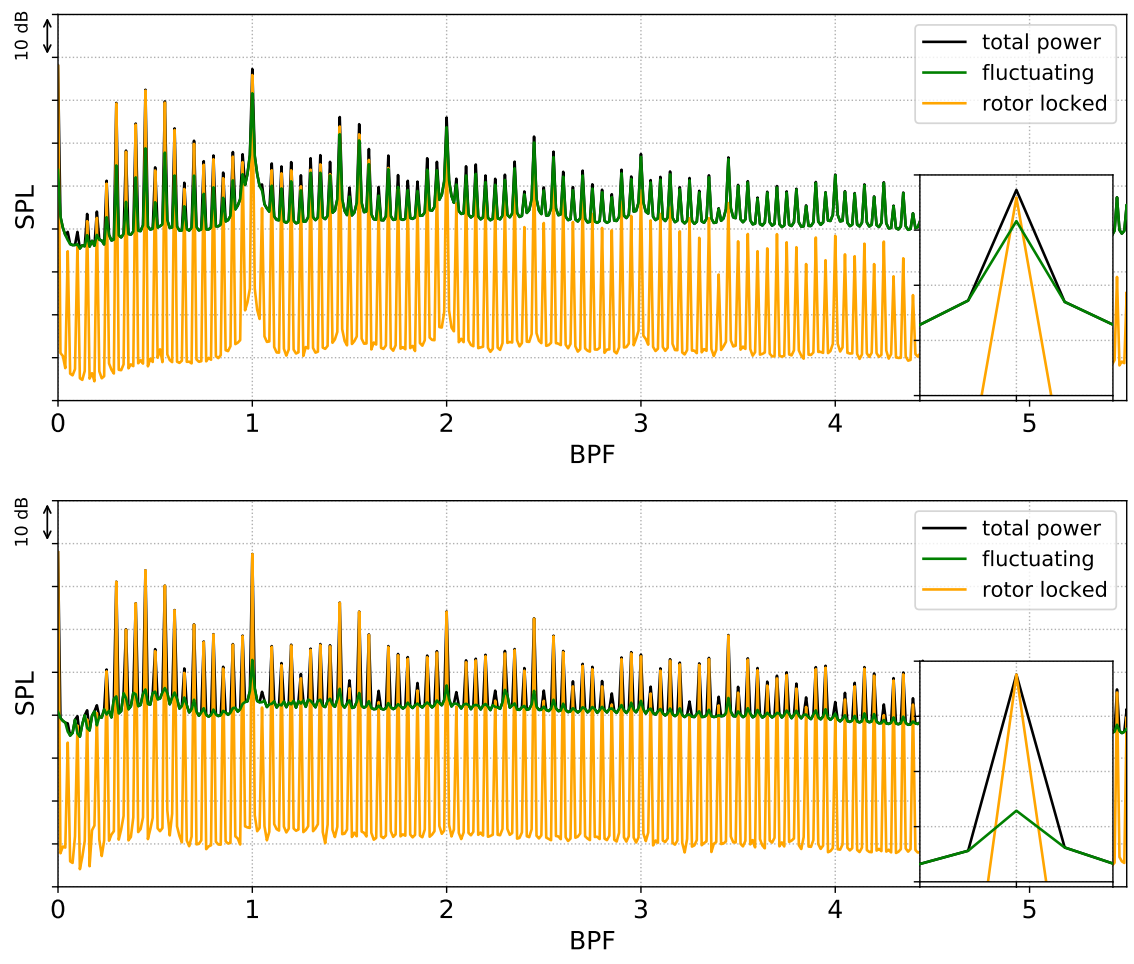

Fig. 11 Frequency spectra of the total power, the rotor locked, and the fluctuating component for the high-speed configuration, averaged over all pressure sensors in the mode detection ring. Top: without, bottom: with the ICD. The inserts show a zoom around the BPF1 peak. 

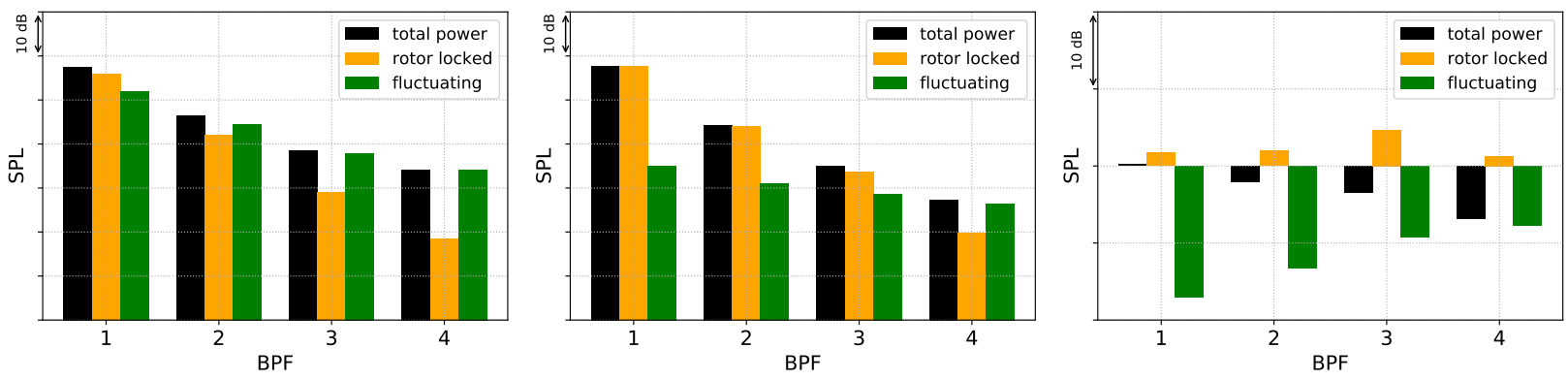

Fig. 12 Sound pressure levels of the BPF and its harmonics of the total power, the rotor locked, and the fluctuating components averaged over all sensors in the mode detection ring for the high-speed configuration. Left: without, centre: with the ICD, right: difference between both configurations.

The rotor alone mode dominates in both cases with the ICD. There are two different reasons for this effect: 1) for the rotor locked component, the amplitudes of neighboring mode orders are significantly reduced and the rotor alone mode is increased, 2) for the fluctuating component, all mode amplitudes are reduced, but the neighboring modes of the rotor alone mode are reduced even more. Without an ICD, the mode amplitudes in the rotor locked component are high because the buzz-saw noise modes that propagate through the non-uniform inflow are increasingly distorted, which increases the levels of neighboring modes [10, 11]. When the buzz-saw modes propagate upstream in the relatively homogeneous flow downstream of an ICD, the flow does not influence the rotor alone mode and its immediate neighbors. The Tyler-Sofrin mode in the rotor locked component of BPF2 (see Fig. 17) increases as well, but it is not significant because the strong buzz-saw noise modes dominate the sound field. In the case with an ICD, only one dominant mode remains and causes a very directed sound radiation into the far field, while the far-field directivity in the configuration without an ICD is relatively homogeneous because of the high number of modes at almost equal strength. The inter-mode coherence for the fluctuating components is shown in Fig. 18 with respect to the rotor alone mode at BPF1 and BPF2. Without the ICD, significant values of the coherence are detected for the neighboring modes. For BPF2, the range of modes that are partly coherent with the rotor alone mode extends over seven neighboring mode orders. The mode coherence distribution exhibits a similar shape as the mode amplitude distribution of the rotor locked components, indicating that the individual modes of the fluctuating components are affected in the same way by the non-uniform inflow as the rotor locked buzz-saw noise mode. The installation of the ICD reduces, again, the inter-mode coherence to negligible values.

\section{Conclusions and outlook}

A cyclostationary analysis of acoustic data measured in a fan test facility with and without an ICD shows that the installation of an inflow control device (ICD) improves the flow quality and leads to a different distribution of power between the rotor synchronous and the fluctuating components of the sound field. In configurations where the fluctuating component dominates, an ICD can reduce the total power at the BPF and its harmonics by substantially reducing the 

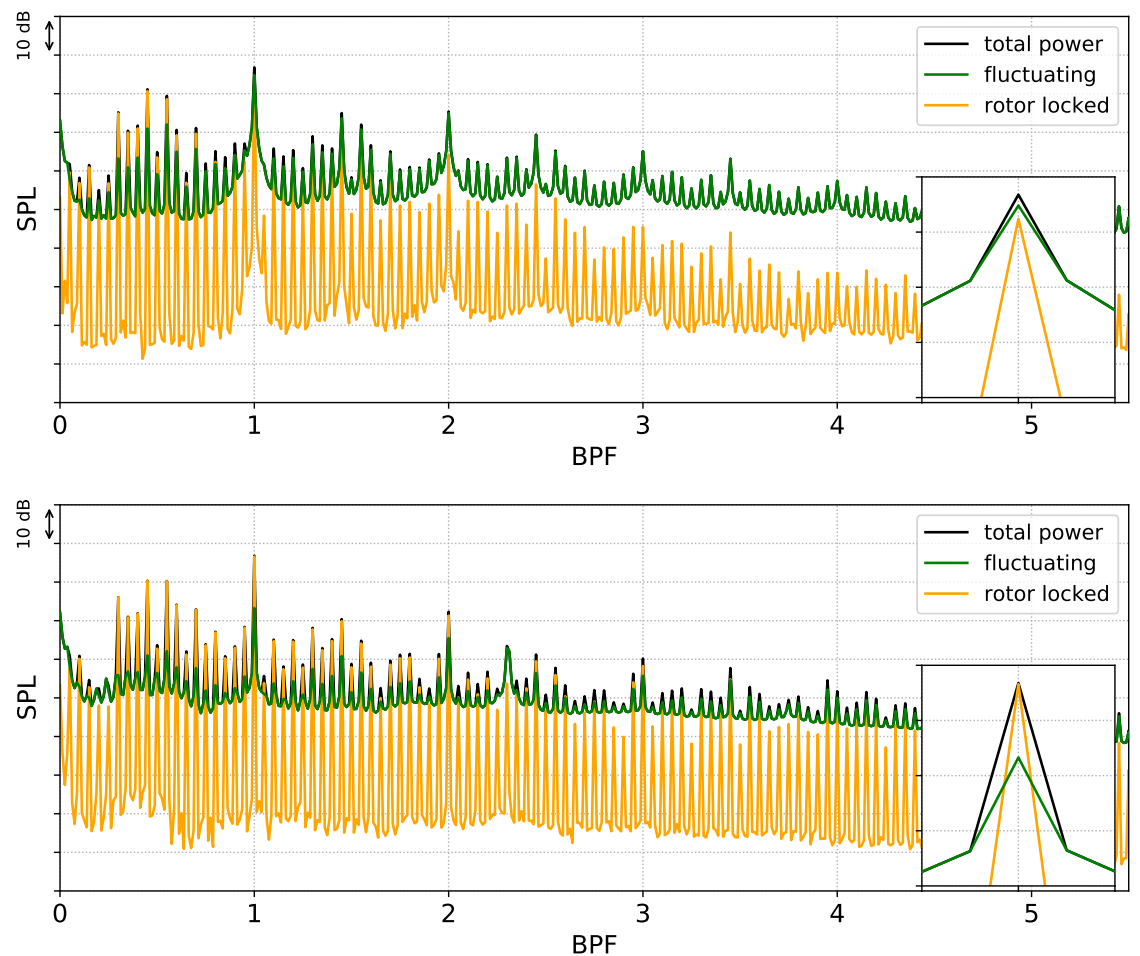

Fig. 13 Frequency spectra of the total power, the rotor locked, and the fluctuating component for the high-speed configuration, averaged over all microphones in the far-field microphone array. Top: without, bottom: with the ICD. The inserts show a zoom around the BPF1 peak.
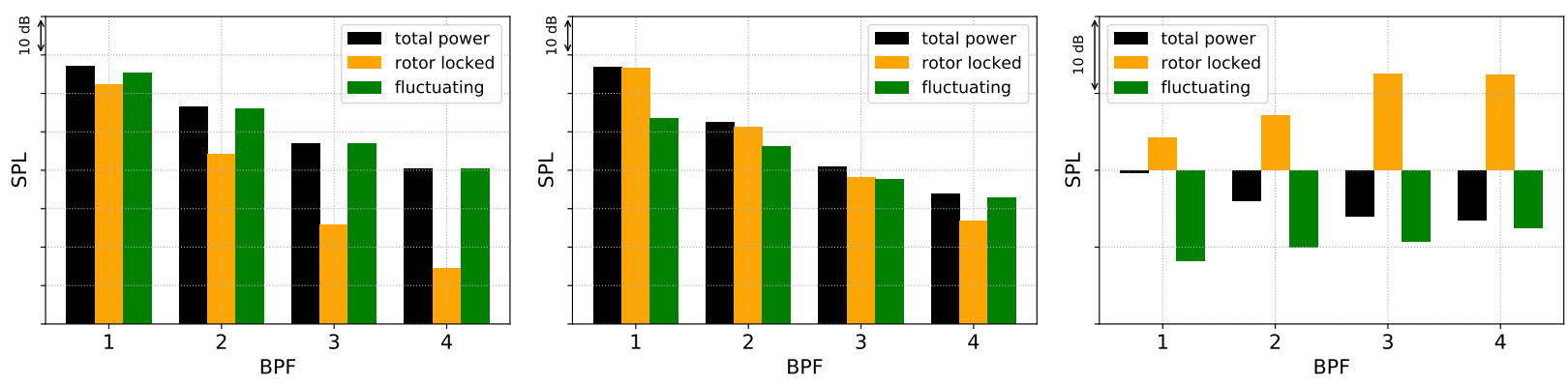

Fig. 14 Sound pressure levels of the BPF and its harmonics of the total power, the rotor locked, and the fluctuating components averaged over the forward arc of the far-field microphones for the high-speed configuration. Left: without, centre: with the ICD, right: difference between both configurations. 

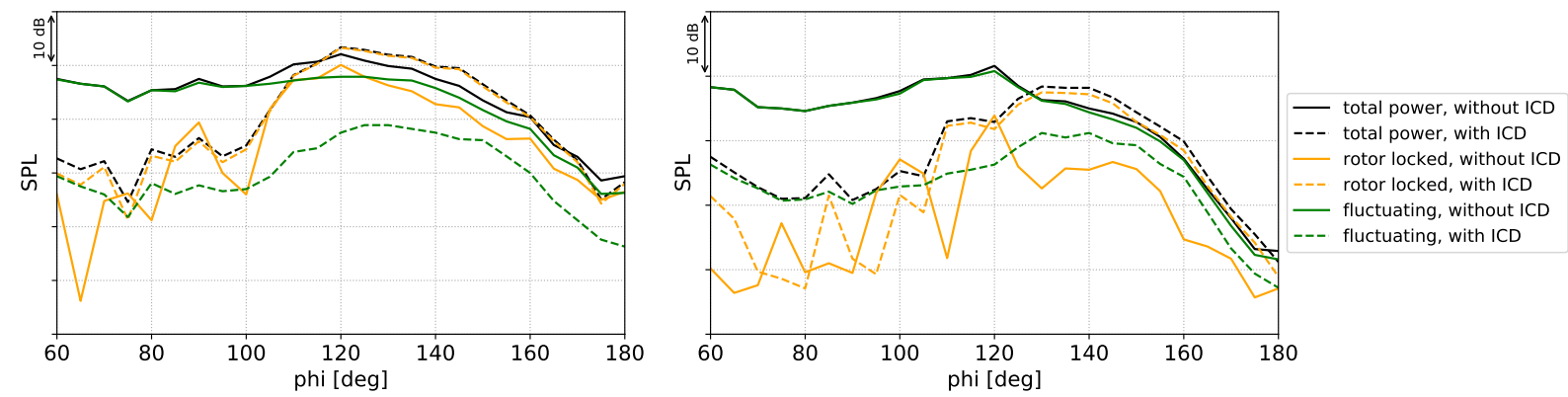

Fig. 15 Far-field directivity of the total power, the rotor locked, and the fluctuating components at the BPF1 (left) and BPF2 (rigth) for the high-speed configuration with and without an ICD. $180^{\circ}$ indicates the direction of flight.
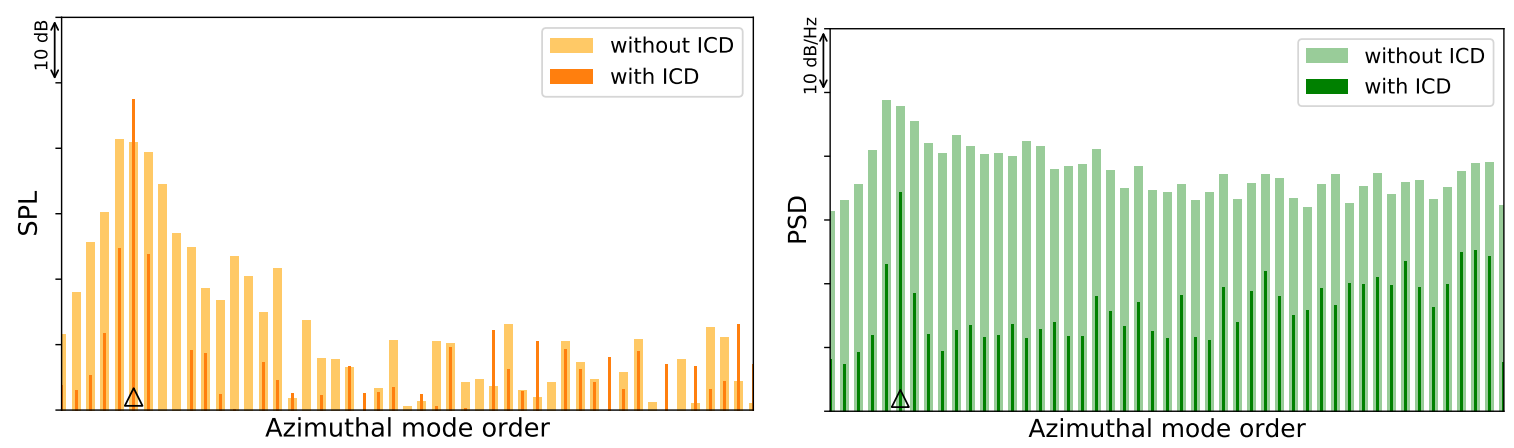

Fig. 16 Comparison of the mode distribution of the rotor locked (left) and the fluctuating (right) component at BPF1 for the high-speed configuration with and without the ICD. The black, hollow triangle marks the rotor alone mode. 

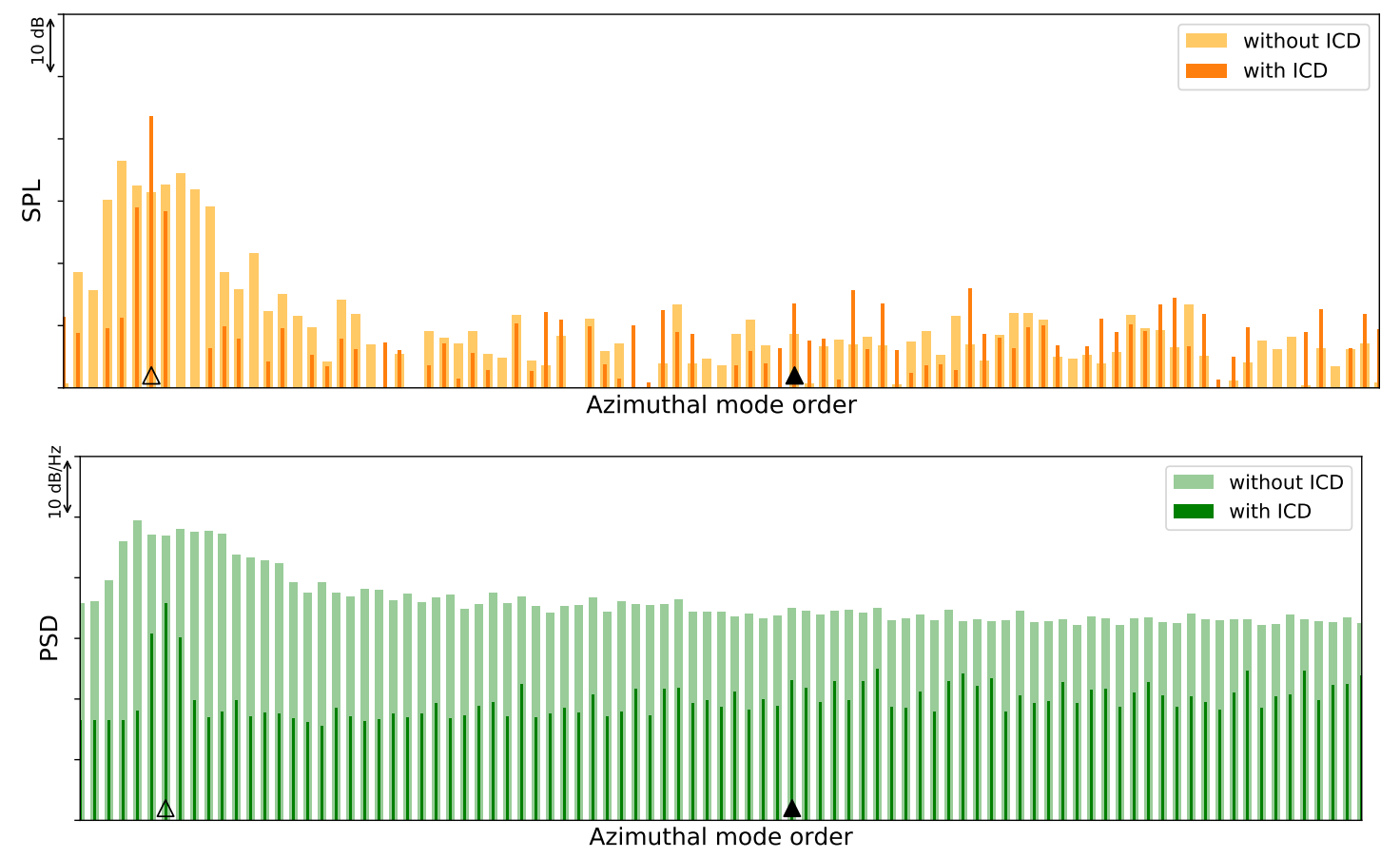

Fig. 17 Comparison of the mode distribution of the rotor locked (top) and the fluctuating (bottom) component at BPF2 for the high-speed configuration with and without the ICD.The black, hollow triangle marks the rotor alone mode and the black, filled triangle the Tyler-Sofrin mode, respectively.
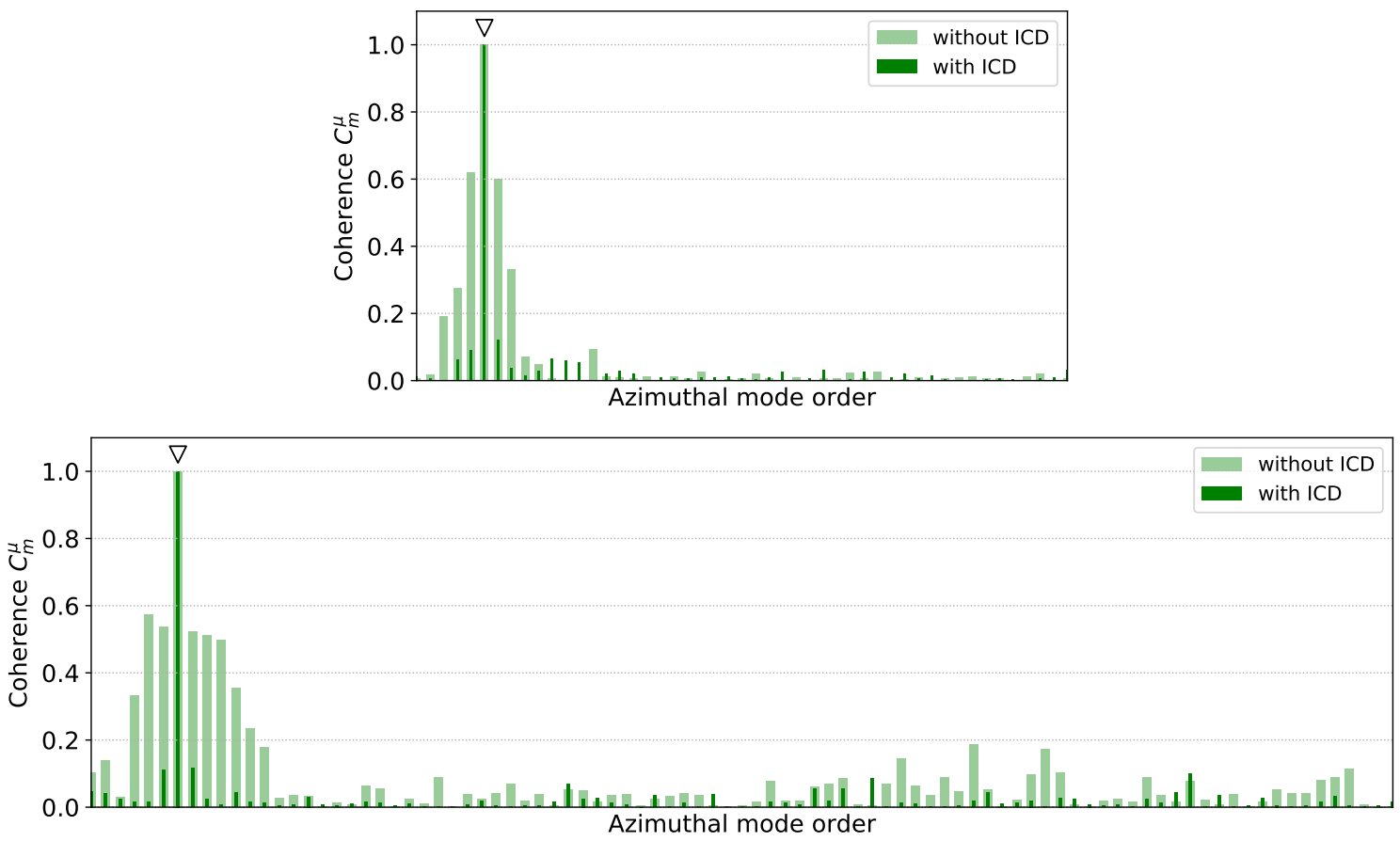

Fig. 18 Comparison of the mode coherence distribution of the fluctuating components w.r.t. the rotor alone mode (indicated by the black, hollow triangle) at BPF1 (top) and BPF2 (bottom) for the low-speed configuration with and without the ICD. 
energy of the fluctuations. The effect of the ICD on the rotor locked power is not as strong. The balance between the rotor locked and the fluctuating component at the BPF and its harmonics changes in favor of the rotor locked component because the energy of the fluctuating component is substantially reduced. The Tyler-Sofrin mode can be stabilized to the extent that it becomes the only dominant mode, if it is cut-on. In configurations where the rotor coherent power dominates the spectrum, i.e. for high, supersonic blade tip speeds with strong tones at the BPF, its harmonics, and buzz-saw noise modes, the ICD reduces the fluctuations and stabilizes the rotor alone mode. The effect of the ICD on the total acoustic energy is relatively small, because the total power is dominated by the rotor locked component.

The far-field measurements at low speed show that the ICD has a relatively small effect on the directivity pattern of the total power and the fluctuating components. At high, supersonic blade tip speeds, where the total power is dominated by the rotor locked component over a wide range of polar angles, the effect on the directivity is more pronounced. While the directivity of the total power measured without an ICD is relatively smooth, the radiation peaks of the BPF1 and BPF2 are shifted forward in the direction of flight when an ICD is installed.

A mode analysis of the unsteady pressure field in the duct shows that the ICD changes the amplitude distribution between the azimuthal modes. In configurations with lower, subsonic shaft speeds, the Tyler-Sofrin mode is stabilized and the bulk of the acoustic energy concentrated in relatively few modes when an ICD is installed.

The results of the analysis suggest that without the ICD, the spread of energy into neighboring mode orders of the Tyler-Sofrin modes is caused by refraction effects when the sound waves propagate through non-uniform flow. In this situation, the azimuthal mode orders do not represent the physical eigenmodes correctly, which could lead to misinterpretations of the mode energy distribution.

An ICD can stabilize the components that are synchronous with the rotor, and it can reduce the random contribution substantially. When the blade tip speed of the fan is subsonic, the fluctuating component is much stronger than the rotor locked component and dominates the sound field at the BPF and its harmonics. In this case, an ICD effectively reduces the noise level at the blade passing frequency by substantially reducing the fluctuations. With the ICD, the energy remains concentrated within the frequency bands of the BPF harmonics. Without the ICD, we observe a spectral broadening at the bases of the BPF peaks, similar to the haystacking effect. The exchange of energy between neighboring bands can be caused either by sound generated by the interaction of turbulence with the rotor or by propagation effects when the sound waves that propagate upstream out of the duct interact with the turbulent boundary layer and ingested turbulence. Which effect dominates cannot be answered on the basis of the data presented here, but the fan BPF levels increase strongly when no ICD is present.

In configurations with supersonic fan tip speeds, the dominating noise source mechanism is the rotor alone noise, which is locked to the rotational speed. The contribution of the fluctuating component to the total power is relatively low. The levels of the fluctuating components and the spectral broadening are reduced by the ICD, however their energy content is much lower than that of the rotor locked modes. Consequently, the reduction of the BPF peak levels, when an 
ICD is installed, is relatively weak. The analysis of inter-mode coherence shows that without an ICD, neighboring modes are partly coherent with the rotor alone mode. This indicates a distortion of the mode shape through refraction by the non-uniform inflow, similar as for the low engine speed. With an ICD, the inter-mode coherence is negligible in the fluctuating components.

The cyclostationary analysis relates steady, spatially fixed inflow distortions (e.g. the velocity deficit in the wakes of upstream installations or the wakes of sensors mounted in the inlet duct) to the rotor locked component of the spectrum. The spectrum of the fluctuating component is influenced by instationary, stochastically fluctuating distortions like turbulent eddies or fluctuating structures like ground vortices, whose fluctuation frequency is generally not linked to the rotor speed.

Both types of distortions, steady and fluctuating, increase the BPF levels. The cyclostationary analysis helps to better understand the changes to the acoustic field when inflow distortions are reduced by an ICD. This technique can be used to investigate the specific mechanisms that increase the BPF levels by applying it to more data from fan test facilities and full scale engine tests. For future studies, the acoustic data should be coupled with aerodynamic data from turbulence and flow profile measurements in order to achieve an even better insight into the physical mechanisms.

\section{Acknowledgements}

The research leading to these results has received funding from the German Federal Ministry for Economic Affairs and Energy (BMWi) in the framework of the Luftfahrtforschungsprogramm (LuFo) projects LisT under the grant agreement 20T1307B and MAMUT under the grant agreement number 20T1524C. The authors thank Rolls-Royce for their support and the permission to use the data and AneCom AeroTest GmbH for the support of the experiments.

\section{References}

[1] Measurement of far field noise from gas turbine engines during static operation, sep 1995. https://doi.org/10.4271/ARP1846.

[2] Methods of Controlling Distortion of Inlet Airflow During Static Acoustical Tests of Turbofan Engines and Fan Rigs, nov 2007. https://doi.org/10.4271/AIR1935.

[3] Atvars, Y., and Rogers, D., "The development of inflow control devices for improved simulation offlight noise levels during static testing of a HBPR turbofan engine," 6th Aeroacoustics Conference, American Institute of Aeronautics and Astronautics, 1980. https://doi.org/10.2514/6.1980-1024.

[4] Rogers, D., and Ganz, U., "Aerodynamic assessment of methods to simulate flight inflow characteristics during static testing," 6th Aeroacoustics Conference, American Institute of Aeronautics and Astronautics, 1980. https://doi.org/10.2514/6.1980-1023.

[5] Woodward, R. P., and Glaser, F. W., "Effect of Inflow Control on Inlet Noise of a Cut-on Fan,” AIAA Journal, Vol. 19, No. 3, 1981, pp. 387-392. https://doi.org/10.2514/3.50960 
[6] Preisser, J. S., and Chestnutt, D., "Flight effects on fan noise with static and wind-tunnel comparisons," Journal of Aircraft, Vol. 21, No. 7, 1984, pp. 453-461. https://doi.org/10.2514/3.44993,

[7] Gliebe, P. R., "Analytical Study of the Effects of Wind Tunnel Turbulence on Turbofan Rotor Noise," Journal of Aircraft, Vol. 18, No. 10, 1981, pp. 818-825. https://doi.org/10.2514/3.57567.

[8] Chestnutt, D., "Flight Effects on Fan Noise," NASA Conference Publication 2242, NASA, 1982. https://ntrs.nasa.gov/archive/nasa/casi.ntrs.nasa.gov/19830002613.pdf.

[9] Adkins, R., "Noise Reduction From Engine Tests at Airports," Volume 2: Turbo Expo 2002, Parts A and B, ASMEDC, 2002. https://doi.org/10.1115/GT2002-30138.

[10] Sofrin, T., and Cicon, D., "Ducted fan noise propagation in non-uniform flow. II - Wave equationsolution," 11th Aeroacoustics Conference, American Institute of Aeronautics and Astronautics, 1987. https://doi.org/10.2514/6.1987-2702.

[11] Moreau, A., and Guérin, S., "Experimental Validation of an Analytical Prediction Model for Fan Buzz-Saw Noise," Proceedings of ASME Turbo Expo, 2020.

[12] Staggat, M., Moreau, A., and Guérin, S., "Analytical prediction of boundary layer ingestion noise for an integrated turbofan," 26th International Congress on Sound and Vibration (ICSV), 2019.

[13] Joseph, P., and Parry, A., "Rotor/wall boundary-layer interaction broadband noise in turbofan engines," 7th AIAA/CEAS Aeroacoustics Conference and Exhibit, American Institute of Aeronautics and Astronautics, 2001. https://doi.org/10.2514/6.20012244.

[14] Ganz, U. W., “Analytical Investigation of Fan Tone Noise Due to Ingested Atmospheric Turbulence,” NASA Contractor Report 3302, Aug. 1980. https://ntrs.nasa.gov/api/citations/19800021654/downloads/19800021654.pdf.

[15] Siller, H., Holland, K., Böhning, P., Arnold, F., and Kempton, A., "Novel Methods for Acoustic Indoor Measurements and Applications in Aero-Engine Test Cells," 6th Berlin Beamforming Conference, 2006. Paper BeBeC-2006-04.

[16] Funke, S., Siller, H. A., Hage, W., and Lemke, O., "Microphone-array measurements of a Rolls-Royce BR700 series aeroengine in an indoor test-bed and comparison with free-field data," 20th AIAA/CEAS Aeroacoustics Conference, Atlanta, Georgia, USA, 2014. https://doi.org/10.2514/6.2014-3070.

[17] Sturm, M., and Carolus, T., "Large Scale Inflow Distortions as a Source Mechanism for Discrete Frequency Sound from Isolated Axial Fans," 19th AIAA/CEAS Aeroacoustics Conference, American Institute of Aeronautics and Astronautics, 2013. https://doi.org/10.2514/F6.2013-2105.

[18] Rademaker, E. R., Sijtsma, P., and Tester, B. J., "Mode detection with an optimised array in a model turbofan engine intake at varying shaft speeds,” Tech. Rep. NLR-TP-2001-132, 2001. https://arc.aiaa.org/doi/pdf/10.2514/6.2001-2181.

[19] Köhler, W., "The Influence of the TCS on the Circumferential Mode Distribution in the Inlet of a Fanrig (UFFA)," 2012, pp. 1813-1822. https://doi.org/10.1115/GT2012-69762. 
[20] Antoni, J., "Cyclostationarity by examples,” Mechanical Systems and Signal Processing, Vol. 23, No. 4, 2009 , pp. 987 - 1036. https://doi.org/10.1016/j.ymssp.2008.10.010.

[21] Behn, M., Pardowitz, B., and Tapken, U., "Separation of tonal and broadband noise components by cyclostationary analysis of the modal sound field in a low-speed fan test rig," FAN 2018 conference, Darmstadt, 2018.

[22] Sijtsma, P., and Brouwer, H., "Deconvolution of azimuthal mode detection measurements," Journal of Sound and Vibration, Vol. 422, 2018, pp. 1-14. https://doi.org/10.1016/j.jsv.2018.02.029.

[23] Behn, M., and Tapken, U., "Investigation of Sound Generation and Transmission Effects Through the ACAT1 Fan Stage using Compressed Sensing-based Mode Analysis," 25th AIAA/CEAS Aeracoustics Conference, Delft, The Netherlands, 2019. https://doi.org/10.2514/6.2019-2502. 